\title{
Pricing Strategy versus Heterogeneous Shopping Behavior under Market Price Dispersion
}

\author{
Francisco Álvarez, ${ }^{1}$ José-Manuel Rey, ${ }^{2,3}$ and Raúl G. Sanchis ${ }^{4,5}$ \\ ${ }^{1}$ Department of Economics, Universidad Complutense de Madrid, Madrid, Spain \\ ${ }^{2}$ Department of Economic Analysis, Universidad Complutense de Madrid, Madrid, Spain \\ ${ }^{3}$ Department of Psychology, Harvard University, Cambridge, MA, USA \\ ${ }^{4}$ Department of Economics, ICADE, Universidad Pontificia Comillas, Madrid, Spain \\ ${ }^{5}$ Instituto Complutense de Estudios Internacionales (ICEI), Universidad Complutense de Madrid, Madrid, Spain
}

Correspondence should be addressed to José-Manuel Rey; j-man@ccee.ucm.es

Received 8 August 2016; Accepted 10 November 2016

Academic Editor: R. Company

Copyright ( 2016 Francisco Álvarez et al. This is an open access article distributed under the Creative Commons Attribution License, which permits unrestricted use, distribution, and reproduction in any medium, provided the original work is properly cited.

\begin{abstract}
We consider the ubiquitous problem of a seller competing in a market of a product with dispersed prices and having limited information about both his competitors' prices and the shopping behavior of his potential customers. Given the distribution of market prices, the distribution of consumers' shopping behavior, and the seller's cost as inputs, we find the computational solution for the pricing strategy that maximizes his expected profits. We analyze the seller's solution with respect to different exogenous perturbations of parametric and functional inputs. For that purpose, we produce synthetic price data using the family of Generalized Error Distributions that includes normal and quasiuniform distributions as particular cases, and we also generate consumers' shopping data from different behavioral assumptions. Our analysis shows that, beyond price mean and dispersion, the shape of the price distribution plays a significant role in the seller's pricing solution. We focus on the seller's response to an increasing diversity in consumers' shopping behavior. We show that increasing heterogeneity in the shopping distribution typically lowers seller's prices and expected profits.
\end{abstract}

\section{Introduction}

More often than not, economic agents must operate in the market under lack of relevant information. Pricing is the primary key strategic variable for any profit maximizing seller with some market power. Each seller sets his own pricetypically different across sellers-and each buyer searches for the lowest price while having a limited capacity to visit shops and learn different prices.

In this paper we adopt the point of view of a single seller and analyze his optimal pricing policy under limited knowledge both about its competitors' prices and the shopping behavior of the typical buyer. The seller has overall information about rival prices represented by a continuous probability distribution $\mathscr{F}$, which is nondegenerate. This fact is referred to in the literature as market price dispersion. Additionally, the seller has an idiosyncratic lower bound $c$ : he is not willing to sell at any price below $c$. The parameter $c$ can be interpreted as the seller's unitary production cost, assuming linear production technology. More generally, $c$ represents the seller's valuation of not selling. Also, we will consider a single representative buyer, who eventually visits a (random) number of shops $n$ from a total sample of size $N$ that has been previously drawn from $\mathscr{F}$. Notice that considering a representative consumer is not a restrictive condition for our analysis, since the relevant decision variable for the seller is the price he should offer to a buyer visiting his shop. The rival prices observed by the buyer are private information; the seller only knows that prices are drawn from $\mathscr{F}$.

The basic setting above can be framed within the literature of price dispersion in economics, business, or marketing. A first issue is why price dispersion should exist in a market for a homogeneous good. Classical economic theory postulates 
the "law of one price" since the seminal competition model by Bertrand [1]. If buyers look at all prices, technologies are linear, and the good on sale is homogeneous, only the shop offering the lowest price operates in the market. However, evidence from decades of empirical studies denies the law of one price, see, for example, the exhaustive survey by Baye et al. [2] or, more recently, the systematic study by Kaplan and Menzio [3]. Stigler [4] introduced search costs on the buyer's side: if each additional price observation is costly, buyers will generally check only a subset of all existing prices. Later research by Burdett and Judd [5] completed the picture by showing that, under the existence of search costs, buyers will only check a subcollection of all existing prices, which in turn induces some price dispersion among sellers.

While theoretical economics may explain why price dispersion occurs-justifying the underlying assumptions in this paper-there is still the issue of how to behave under price dispersion. The optimal behavior of buyers under search cost has been analyzed in the literature with the focus on characterizing a (nondegenerate) price distribution that conforms an equilibrium. Here we opt for a more practical view: a seller in the market must define a pricing policy even off equilibrium. This angle turns out to be more relevant in real markets with large and persistent disequilibria.

A key feature in our pricing model is the fact that shopping behavior is diverse (or heterogeneous), that is, the number of sellers visited to make their purchase varies across consumers. Alternatively, a representative consumer selects the number of sellers to visit according to some (nondegenerate) probability distribution $\Phi$. The seller's problem thus consists of selecting the price that maximizes his expected profit in an environment of price dispersion and, also, of diverse shopping behavior.

In the proposed model, a basic pricing policy maps an input quadruple to the price $p^{*}$ that yields maximal expected profits, namely, $(c, \mathscr{F}, \Phi, N) \rightarrow p^{*} \in[c, \infty)$, where $c$ is the seller's unit cost, $\mathscr{F}$ is the market price distribution, $\Phi$ is the shopping distribution, that is, the distribution of the number of shops which are visited by a representative consumer, and $N$ is the size of the sample of prices.

We solve numerically the seller's problem for different costs, price distributions, and shopping distributions. We consider $\mathscr{F}$ to be a Generalized Error Distribution (GED hereafter), which allows us to consider a wide variety of price distributions by changing the first, second, and higher order moments. The GED family includes normal and quasuniform distributions as particular cases. We are particularly interested in the role of the shape of $\mathscr{F}$ in the seller's solution. Apparently, the question whether the shape of the price distribution does or does not condition the optimal pricing behavior has not been considered in the literature. Furthermore, it was shown in Alvarez et al. [6] that changes in the first two moments of $\mathscr{F}$, but not in its shape, affect the consumer's shopping behavior (determined via efficient time allocation). We will show below that this is not the case for the seller's pricing problem.

We assume that $\Phi$ is a discrete distribution defined on the set $\{2, \ldots, N\}$, so that a representative consumer selects a priori a sample of $N$ shops and then visits a number $n$ of them with probability $\Phi(n)$ (to keep notation simple we will use $\Phi$ interchangeably for the shopping distribution and its probability mass function). This amounts to consider that the consumer's behavior is perceived as probabilistic by the seller. In turn, this probabilistic perception might be exclusively due to the seller's lack of knowledge or, alternatively, it might be that the buyer's behavior is intrinsically probabilistic. Our main finding here is that, once a buyer goes shopping, more diversity in his shopping behavior, or, alternatively, more uncertainty in the knowledge of the seller about the consumer's behavior, entails lowering prices and expected profits.

The pricing model above can be implemented in real markets once accurate estimates of market prices and consumer behavior are available. Estimates of the two basic inputs, $\mathscr{F}$ and $\Phi$, of the pricing model above can in principle be obtained for specific markets. Price histograms for a large number of goods and markets can be obtained from available consumer data sets and they can be typically fitted by some $\mathscr{F}$ within the GED family, as shown by Kaplan and Menzio [3]. Shopping data required to estimate $\Phi$ are not commonly available in the literature or public databases. Yet, some studies on choice overload can provide partial information about $\Phi$ (see, e.g., the references in the survey by Scheibehenne et al. [7]).

The manuscript is organized as follows. In Section 2 we describe the problem of a seller maximizing his expected profits in an environment of dispersed prices, which we solve numerically as explained in Section 3. In Section 4 we discuss the results of the numerical analysis. We conclude in Section 5 with final remarks.

\section{The Seller's Problem}

We adopt the point of view of a seller (or a firm) with linear production technology that must decide the price of his product in a market with an indeterminate number of competitors. The main elements of the scenario in which the seller makes his pricing decision are the existence of market price dispersion and imperfect knowledge about rivals' prices and about the shopping behavior of his potential customers.

Specifically, the seller sets his price in a market in which a representative consumer with a fixed demand of, say, one unit, goes shopping. The seller has information about rivals' prices represented by a probability distribution $\mathscr{F}$. The consumer selects a priori $N$ sellers or shops to visit, obtained as a random draw from $\mathscr{F}$. The products offered by the different sellers are indistinguishable for the consumer; that is, the market product is homogeneous. Consequently, he just searches for the lowest price. The consumer eventually observes $n$ price quotes, obtained as a subcollection of the preliminary sample of size $N$.

In general, shopping behavior is diverse, so that a population of consumers is expected to be nonhomogeneous in terms of the number of shops to be visited. In turn, the shopping behavior of a representative consumer can be understood as probabilistic and heterogeneous. This heterogeneity is represented by a distribution $\Phi$ supported on the set $\{2, \ldots, N\}$, so that a consumer will visit $n$ shops with 
probability $\Phi(n)$. We assume that $n>1$ in the shopping behavior: if $n=1$, the seller acts as a monopolist regardless of its rivals prices (regardless of $\mathscr{F}$ ) since the consumer only checks one price. From the seller's perspective, this probability distribution also reflects his uncertainty about the type of consumer who will visit his shop: he just knows that a consumer that visits $n$ shops will show up with probability $\Phi(n)$.

The seller's problem thus consists of finding the mapping $(c, \mathscr{F}, \Phi, N) \rightarrow p^{*}$, where $p^{*}$ is the optimal price; that is, $p^{*}$ maximizes the seller's expected profits. Expected profits are defined by

$$
\pi(p)=(p-c) S(p),
$$

where $S(p)$ is the probability of success in selling at price $p$. Specifically, $S(p)$ is the probability of making the sale at price $p$, given that a consumer will buy at the lowest price after having visited a number of $n$ shops, $n=2,3, \ldots, N$, (with probability $\Phi(n)$ ), from a sample of size $N$ obtained from $\mathscr{F}$. Notice that $S(p)$ can be written as

$$
\begin{aligned}
S(p) & =\sum_{n=2}^{N} \Phi(n) \frac{\left(\begin{array}{c}
N-1 \\
n-1
\end{array}\right)}{\left(\begin{array}{c}
N \\
n
\end{array}\right)}(1-F(p))^{n-1} \\
& =\sum_{n=2}^{N} \Phi(n) \frac{n}{N}(1-F(p))^{n-1},
\end{aligned}
$$

where $F$ is the cumulative distribution function of the distribution $\mathscr{F}$. Now, given a price $p$, the first term in equation (1) is simply the mark-up, the difference between price and unitary cost. The chances $S(p)$ that the seller earns his mark-upmaking the sale-competing in price with other $N-1$ sellers require that (i) the consumer visits the seller's shop given that he will visit $n$ shops out of $N$, and (ii) the seller's price is the lowest among those $n$ prices checked by the consumer. The second factor in each term of the sum in (2) corresponds to (i), whereas the third factor corresponds to (ii). Given that the consumer will visit $n$ shops with probability $\Phi(n),(2)$ gives the probability of making the sale at price $p$. In the case that any of the events (i) or (ii) does not occur, $S(p)=0$, and consequently the seller makes zero profits, so that (1) can be interpreted as expected profit.

Notice that the seller faces a trade-off when choosing his price: increasing the price (the mark-up) implies increasing effective profits in the case he sells the unit, but also lowering the probability of selling the unit.

Shopping behavior is understood here as the choice of the number $n$ of shops to visit (alternatively, prices to check). Even in the case that the consumer has selected a total number $N$ of shops to visit a priori, he may actually end up visiting a smaller number $n$. The classical literature of search cost (e.g., Stigler [4]) gives a rational explanation for this shopping behavior: assuming that the consumer minimizes her total expected cost-search cost plus expected price to be paid-the optimal $n$ may be typically lower than $N$. Alternatively, the consideration of time as a fundamental constraint affecting consumer's behavior can also explain the rational choice of a number of visits $n$ below $N$. This applies in particular when the total number of shops $N$ is large and the consumer optimally allocates his time among several alternative uses. Indeed, it was shown in Sanchis et al. [8] and Alvarez et al. [6] that assuming that $n$ is determined from the time allocation that maximizes well-being, a consumer might choose not to visit the total number $N$ of available shops.

No particular optimal behavior on the consumer's part will be assumed here. In fact, behavioral scientists claim that consumers' behavior may be far from rational in this kind of shopping environment. The so-called choice overload phenomenon is a focal example in which consumers are worse off when they visit all available shops (see, e.g., Schwartz [9] and Iyengar and Lepper [10]). A consumer who visits all available shops is called a maximizer by social psychologists; otherwise he may be generically called a satisficer [11]. A consumers' population is not expected to be composed by maximizers only, but rather by a mixture of maximizers and different types of satisficers. This mixture is represented in this paper by the shopping distribution $\Phi$ : given a sample of $N$ shops in the market, $\Phi(N)$ is the probability that the representative consumer is a maximizer, whereas $\Phi(n)$, for $2<n<N$, are the probabilities that he is some type of satisficer.

\section{Numerical Analysis: Input Data and Problems under Study}

We follow a numerical approach for the analysis of the seller's problem described above. Given the unitary $\operatorname{cost} c$, a distribution of market prices $\mathscr{F}$, the number $N$ of sampled sellers, and a shopping distribution $\Phi$, a core computational routine must produce a numerical solution to problem (1). Since an explicit solution can only be obtained in very special cases, the numerical analysis becomes essential. We have used $\mathrm{R}$ as a programming environment ( $\mathrm{R}$ Core Team [12]).

We consider the market price distribution $\mathscr{F}$ to be a Generalized Error Distribution GED $(\mu, s, \nu)$, whose parameters are location $(\mu)$, scale $(s)$, and shape $(\nu)$ [13]. GED is a family of symmetrical unimodal distributions with domain in $(-\infty,+\infty)$ whose probability density function is given by

$$
f(p)=\frac{\nu}{2 s \Gamma(1 / \nu)} \exp \left(-\left(\frac{|p-\mu|}{s}\right)^{\nu}\right),
$$

where $\Gamma$ denotes the Gamma function. The parameter $\mu$ locates the mean, the median, and the mode of the distribution. The variance of the distribution depends on both scale $s$ and shape $v$, as follows:

$$
\sigma^{2}:=\operatorname{Var}[p]=\frac{s^{2} \Gamma(3 / \nu)}{\Gamma(1 / \nu)} .
$$

Our choice is due to the fact that GED is a flexible family of distributions that includes Laplace (double exponential) distributions if $v=1$, normal distributions if $v=2$, or quasiuniform distributions when $v$ is large enough. In fact, the GED converges pointwise to a uniform distribution on $(\mu-s, \mu+s)$ as $\nu \rightarrow+\infty$. Several GED probability densities for different shapes are displayed in Figure 2(a).

The skewness of a GED is zero, while the excess of kurtosis depends exclusively on the shape $\nu$. In fact, GEDs with 
shape $v<2$ are leptokurtic. This is a significant case, since distribution of prices for individual goods appears to be leptokurtic in many cases (see Kaplan and Menzio [3]). In the model analysis with respect to shape below both leptokurtic (e.g., Laplace) and platykurtic distributions (e.g., quasiuniform) are considered. We set the mesokurtic case $v=2$ (normal distributions) as the benchmark case below for other analyses where shape is constant.

The solution to our seller's problem can be written in parametric form as a mapping

$$
\left(c,(\mu, s, \nu),(\Phi(n))_{n=2, \ldots, N}, N\right) \longrightarrow\left(p^{*}, \pi^{*}\right),
$$

where $p^{*}$ is the maximum of (1) and $\pi^{*}:=\pi\left(p^{*}\right)$ is the seller's optimal (expected) profit. We are mainly interested in analyzing the response of the output variables $\left(p^{*}, \pi^{*}\right)$ as the diversity of satisficers in the market increases. This amounts to study the seller's solution with respect to suitable variations in $\Phi$ while keeping everything else constant. Notice that a homogeneous population of maximizers can be defined by $\Phi(N)=1$. When the probability mass is spread over the set $\{2, \ldots, n\}$, the heterogeneity of the distribution $\Phi$ increases with respect to the maximizer case $\Phi(N)=1$. It will be useful to characterize the degree of heterogeneity using some sensible parameter. We analyze the effects on price and profits of an increase in shopping heterogeneity which is due to two different sources, in turn controlled by two different parameters. First we consider in Section 4.2.1 uniform spreads over an increasing range of $n$ 's, $n \in$ $\{2, \ldots, K\}$, with $1<K \leq N$, which can be parameterized using the Shannon's entropy of the distribution. Second, in Section 4.2.2, we analyze distributional spreads generated by shopping fatigue that can be parameterized by the probability $\rho$ of not visiting a new shop after having visited a number of them.

As a preliminary study, in Section 4.1, we consider a homogeneous population of maximizers, that is, $\Phi(N)=1$, and analyze the effect of a variation in some basic parameters of the problem on the seller's solution. We are particularly interested in learning whether the seller's solution depends only on the mean and dispersion-typical deviation-of prices or whether the shape of the price distribution also matters.

\section{Results and Discussion}

Initially, we assume a homogeneous population of maximizers, so that $\Phi(N)=1$, and we analyze the seller's response with respect to changes in the cost or in a distributional parameter of prices, while keeping everything else constant except for the market size $N$-the number of available sellers a priori. The price distribution $\mathscr{F}$ is assumed to be GED with shape $v=2$, which corresponds with a normal distribution. The results do not differ if a different GED is considered. We are particularly interested in the effect of the shape of the price distribution on price and profits. Typically, for a set of values of $N$, we compute and compare the seller's solution within a range of equispaced values of each parameter, while keeping the rest of parameters at their benchmark values. The benchmark case and the range of variation considered for
TABLE 1: Benchmark and range of analysis for price distribution parameters, cost, and market size.

\begin{tabular}{lccccc}
\hline & \multicolumn{2}{c}{$\operatorname{GED}(\mu, s, \nu)$} & & Cost & Market size \\
& $\mu$ & $\sigma^{2}$ & $\nu$ & $c$ & $N$ \\
\hline Benchmark & 600 & 125 & 2 & 470.44 & 30 \\
Range of analysis & $575-625$ & $100-150$ & 2 & $425-475$ & $2-30$ \\
\hline
\end{tabular}

TABLE 2: Benchmark case for Section 4.1.

\begin{tabular}{|c|c|c|c|c|c|}
\hline & \multicolumn{3}{|c|}{$\operatorname{GED}(\mu, s, \nu)$} & \multirow{2}{*}{$\begin{array}{c}\text { Competitiveness } \\
\qquad \alpha_{c}\end{array}$} & \multirow{2}{*}{$\begin{array}{c}\text { Market size } \\
N\end{array}$} \\
\hline & $\mu$ & $\sigma^{2}$ & $v$ & & \\
\hline $\begin{array}{l}\text { Shape analysis } \\
\text { (range) }\end{array}$ & 600 & 125 & $1-10$ & 0.15 & $2-30$ \\
\hline
\end{tabular}

each parameter are shown in Table 1. The benchmark value of $c$ in Table 1 corresponds to the price level which is higher than $15 \%$ of all prices.

Assuming a maximizer profile for the representative consumer, it can be checked consistently that an increase in the market size $N$ leads to more competitive prices-that is closer to the unitary cost $c$-whereas an increase in the unitary cost or in the mean price $\mu$ leads to higher prices. In turn, the associated profits shrink as $N$ increases or as $c$ increases, but they grow as $\mu$ increases. These facts account for responses of the seller's solution that could be somehow expected. The corresponding graphs are displayed in Figure 1.

4.1. Shape Variation in Price Distribution. As mentioned above, we are particularly interested in the seller's response with respect to variations in the shape of the price distribution. This is a relevant question from the economic perspective. The use of $\operatorname{GED}(\mu, s, \nu)$ as price distributions is particularly useful here, since the shape $v$ of the distribution can be changed while keeping mean $\mu$ and variance $\sigma^{2}$ constant. Notice that a GED with a given $\sigma^{2}$ can be obtained for any shape $v$ by adjusting the scale parameter $s$ suitably in (4). Again, the representative consumer is a maximizer here.

In order to isolate the shape effect on the seller's solution, the unitary cost $c$ needs to be adjusted so that $F(c)$ remains constant at a certain level $\alpha_{c}$. Such $\alpha_{c}$ represents the fraction of rivals whose costs are lower than the cost of the seller under analysis. It can be interpreted as a competitiveness level, since de facto the seller could not compete in price with $100 \times \alpha_{c}$ percent of other rivals in the market. The competitiveness level will remain invariant for different shapes in the analysis below. Also, the mean and dispersion of the price distribution remain constant. As mentioned above, this can be controlled within the GED family by adjusting the scale parameter $s$ to keep variance fixed as $v$ varies. Table 2 shows the benchmark and the range of variations in shape $v$ and market size $N$.

The typical output of the analysis in this section is displayed in Figure 2(b) for $N=30$. The main message of the analysis is that shape matters; that is, when it comes to choose the optimal price, information about mean and dispersion of the market prices is not enough. A seller should also have an estimate of the shape of the price distribution. Thus, while the total mass of effective competitors (i.e., $100 \times\left(1-\alpha_{c}\right)$ percent of the sellers) is important, also the shape according 


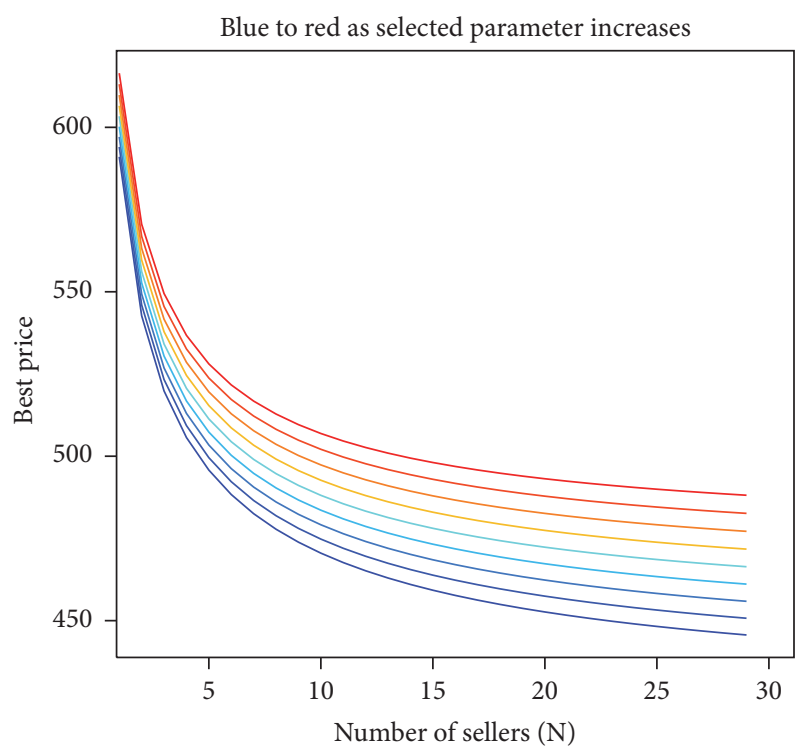

(a) Prices versus $N$ as $c$ increases

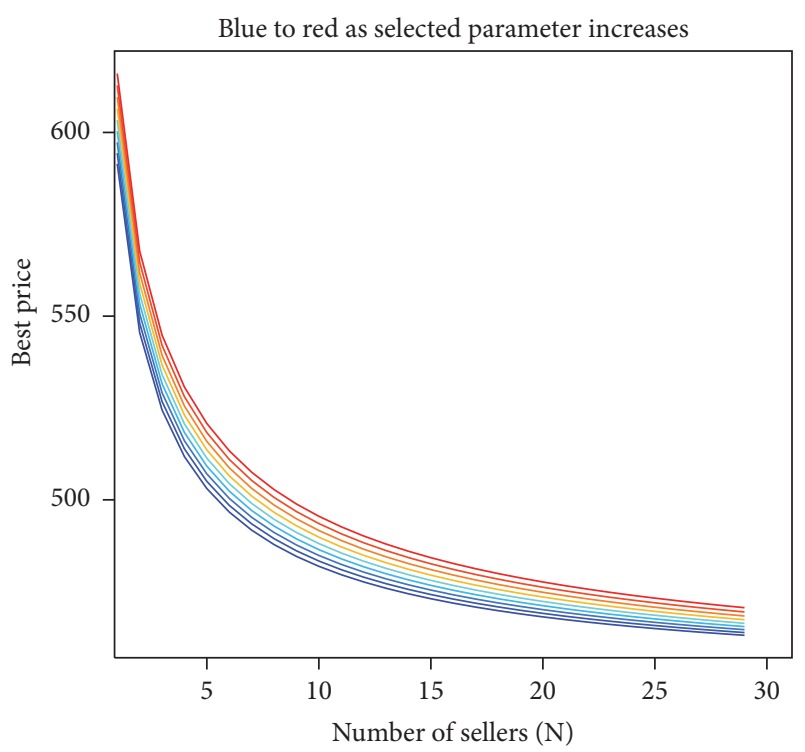

(c) Prices versus $N$ as $\mu$ increases

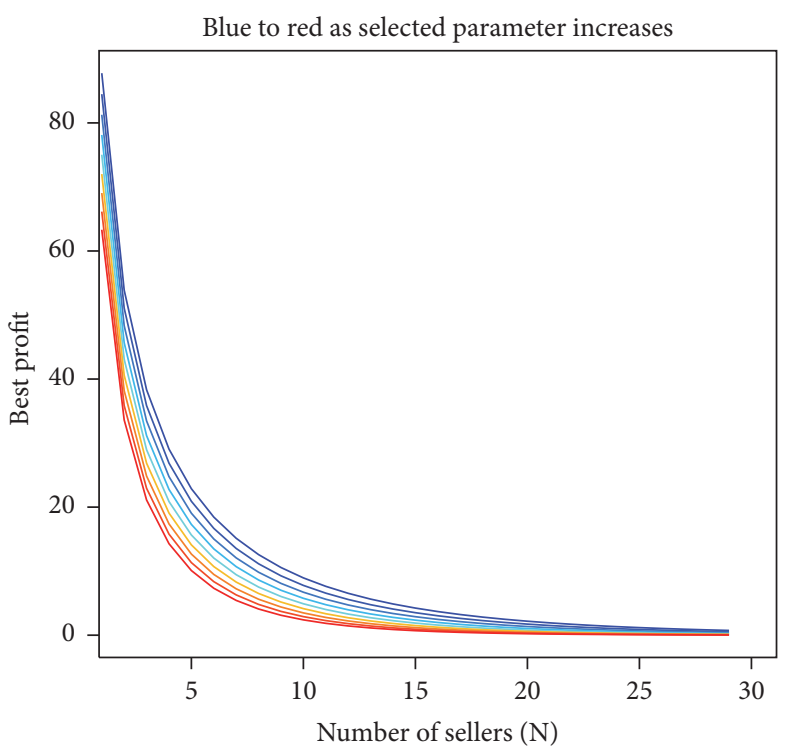

(b) Profits versus $N$ as $c$ increases

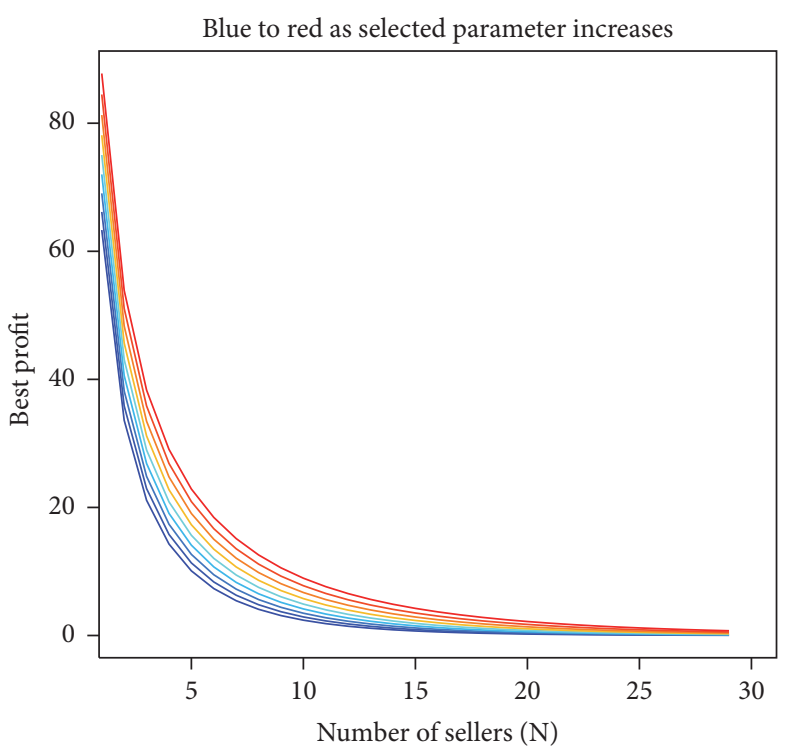

(d) Profits versus $N$ as $\mu$ increases

FIGURE 1: Responses in optimal price and (expected) profits to changes in cost $c$, price mean $\mu$, and market size $N$. From blue to red as cost and price mean increase within the range of analysis and with parameter values as in the benchmark case $\left(\mu=600, \sigma^{2}=125, \nu=2, N=30\right)$; see Table 1.

to which this probability is distributed is relevant for the seller's problem.

The study above suggests that knowledge of mean and dispersion of prices does not suffice to set prices rationally. This is in contrast with the analysis of shopping behavior of consumer-that is their choice of the subsample sizesearching for the lowest price under optimal allocation of their time. The analysis in Alvarez et al. [6] suggests that their optimal choices depend only on the first and second order moments of the (symmetric) price distribution. Therefore, while knowledge of the mean and the dispersion of prices seems sufficient for the consumer to solve his shopping problem-as a by-product of optimal time allocation-it is not enough for the seller to define his pricing policy efficiently.

4.2. Heterogeneity in Shopping Behavior. The seller's response is analyzed next with respect to variations of parameters of the shopping distribution $\Phi$, keeping everything else fixed. We will analyze the effect of increasing the heterogeneity of the shopping distribution, that is, augmenting the variety of consumer's profiles in terms of the number of visited shops. We consider below two ways of increasing heterogeneity in $\Phi$ : in Section 4.2.1 we consider uniform spreads over 


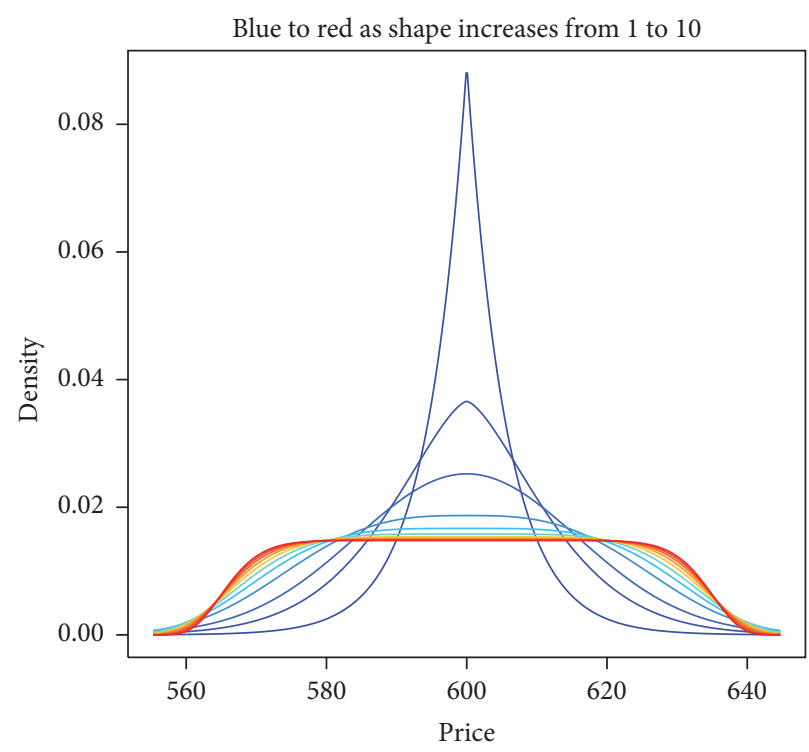

(a) Probability density functions

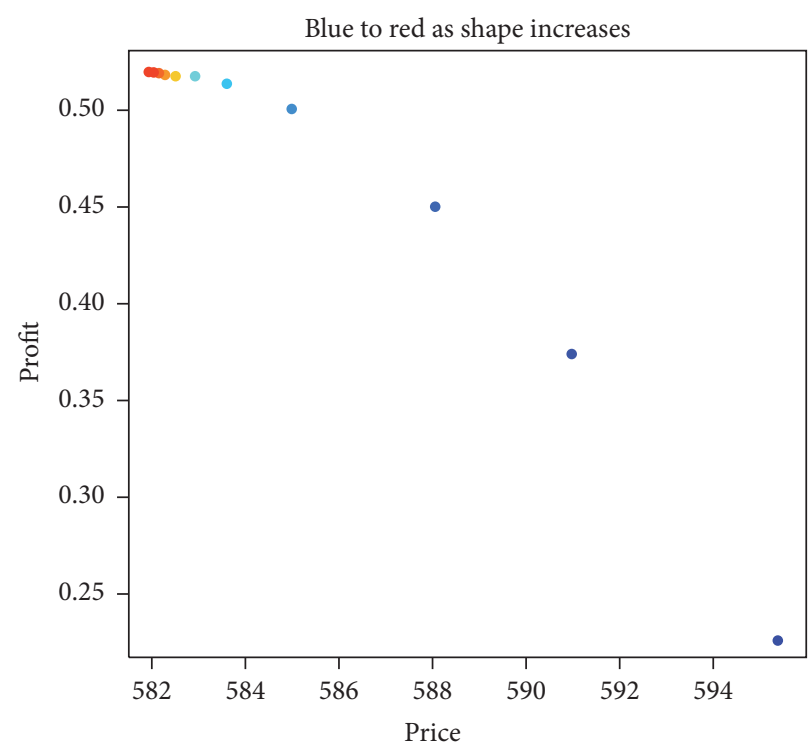

(b) Profits versus prices

FIGURE 2: Probability density functions of prices and output from the analysis in Section 4.1 for GED $(\mu, s, \nu)$ distribution with varying shape (from blue $(\nu=0.5)$ to red $\left(\nu=10\right.$, quasiuniform)) and the rest of parameters as in the benchmark case $\left(\mu=600, \sigma^{2}=125, N=10\right)$; see Table 2.

TABLE 3: Heterogeneous consumer's shopping distributions in Sections 4.2.1 and 4.2.2 and their respective benchmarks.

\begin{tabular}{lccc}
\hline & Shopping distributions & Heterogeneity analysis (range) & Benchmark \\
\hline Spread-of-search (Section 4.2.1) & $\Phi_{K}$ & $K=2,3, \ldots, 30$ & $K=2$ \\
Search fatigue (Section 4.2.2) & $\Phi_{\rho}$ & $\rho=0.05 i, i=1,2, \ldots, 20$ & $\rho=1$ \\
\hline
\end{tabular}

an increasing range of $n$ 's, and in Section 4.2.2 we analyze distributions generated by a fatigue effect on consumer's shopping behavior.

4.2.1. Increasing Spread of Shopping Distribution. We assume here that $\Phi=\Phi_{K}$ is the uniform distribution on the set $\{2,3, \ldots, K\}$, for every integer $2 \leq K \leq N$, that is $\Phi_{K}(n)=$ $(K-1)^{-1}$ for $n \in\{2,3, \ldots, K\}$. The benchmark case is $K=$ 2 ; that is, the representative consumer visits 2 shops. The benchmark values for other relevant parameters are as in Table 1 . We analyze the seller's response with respect to a uniform spread in the number of possible visits; that is, we compute his pricing policy and associated profits when a representative consumer chooses randomly a number $n$ of shops to visit between 2 and $K$, with equal probability. The seller is totally uncertain about the precise number of shops (between 2 and $K$ ) the consumer will eventually visit: any number is equally likely. As $K$ increases, the Shannon's entropy of $\Phi_{K}$, given by $H_{K}=\log (K-1)$, also increases. The entropy $H_{K}$ serves as a measure of the heterogeneity of the distribution and, alternatively, of the uncertainty of the seller with regard to the consumer side of the market (see, e.g., Gray [14]).

Figure 3(b) summarizes the seller's optimal responseprices and associated (expected) profits-as the entropy of the distribution increases. It can be observed that prices and profits decrease as the heterogeneity of the distribution increases. As the demand side of the market becomes more competitive (i.e., an extra shop is equally likely to be visited), the seller's profits shrink.

4.2.2. Search Fatigue in Shopping Behavior. We consider here a behavioral effect as a possible source of the heterogeneity in the shopping distribution. Assume that a representative consumer has selected a sample of $N$ potential sellers, or shops, to visit (i.e., prices to check) from $\mathscr{F}$ and that he will visit at least 2 shops. We further assume that, after having visited any number of shops, there is a probability $0<\rho<1$ of not visiting one more shop. In principle, the fatigue parameter could have memory of the number of shops already visited, that is, $\rho=\rho(n)$. We consider here the simple case in which $\rho$ is constant.

The simple behavioral assumption above produces a shopping probability distribution $\Phi_{\rho}$ with support $\{2,3, \ldots$, $N\}$ if $0<\rho<1$. Indeed, the probability that a representative consumer will visit $n$ shops, given that he knows that a total of $N$ shops is available is given by

$$
\Phi_{\rho}(n)=\frac{\rho(1-\rho)^{n-1}}{1-(1-\rho)^{N-1}},
$$

which is a version of the geometric distribution [13]. Notice that if $\rho=1$ the consumer will visit just 2 shops almost surely, which is the benchmark case considered in Table 3. 


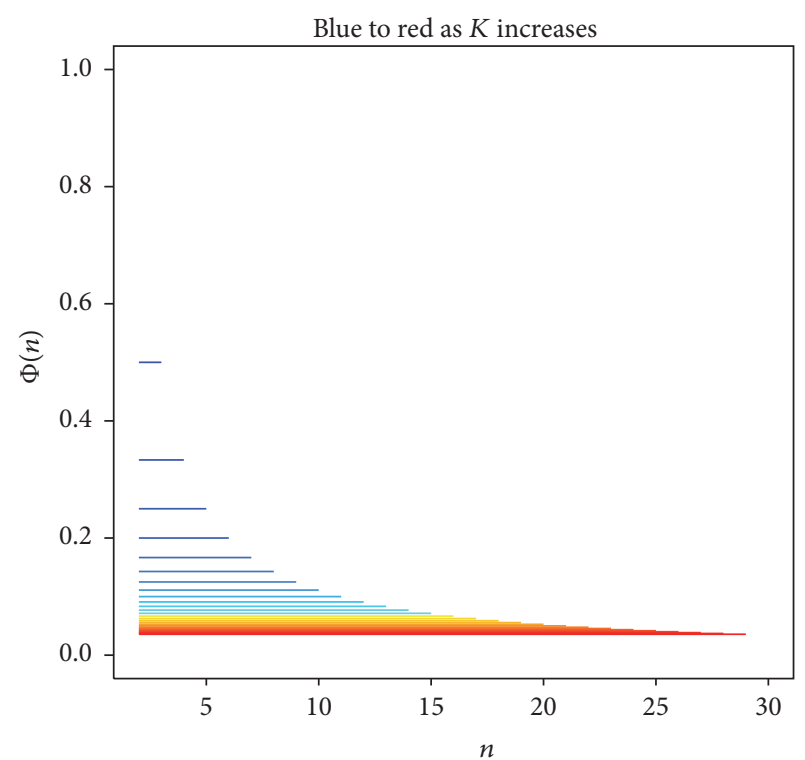

(a) Probability mass functions as the number of visited shops $K$ increases from blue $(K=2)$ to red $(K=30)$

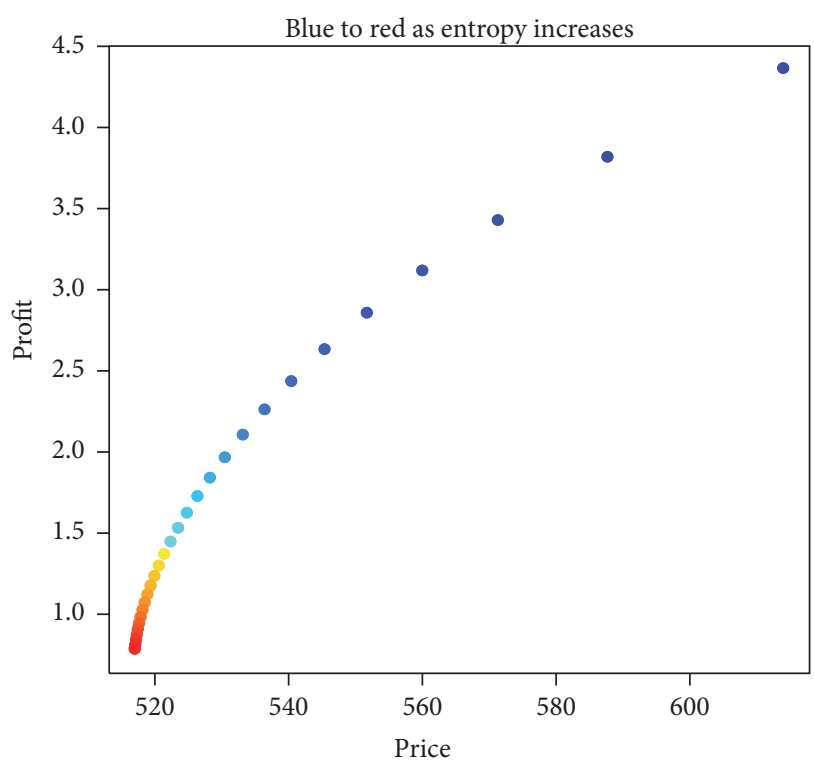

(b) Profits versus prices (optimal) for an increasing spread of the shopping distribution $\Phi_{K}$ as entropy increases from blue $(K=2)$ to red $(K=30)$

FIgURE 3: The seller's solution under a uniform spread of shopping behavior. Main parameters in Table 3 and those used earlier as benchmark $\left(\mu=600, \sigma^{2}=125, \nu=2, \alpha_{c}=0.15, N=30\right)$.

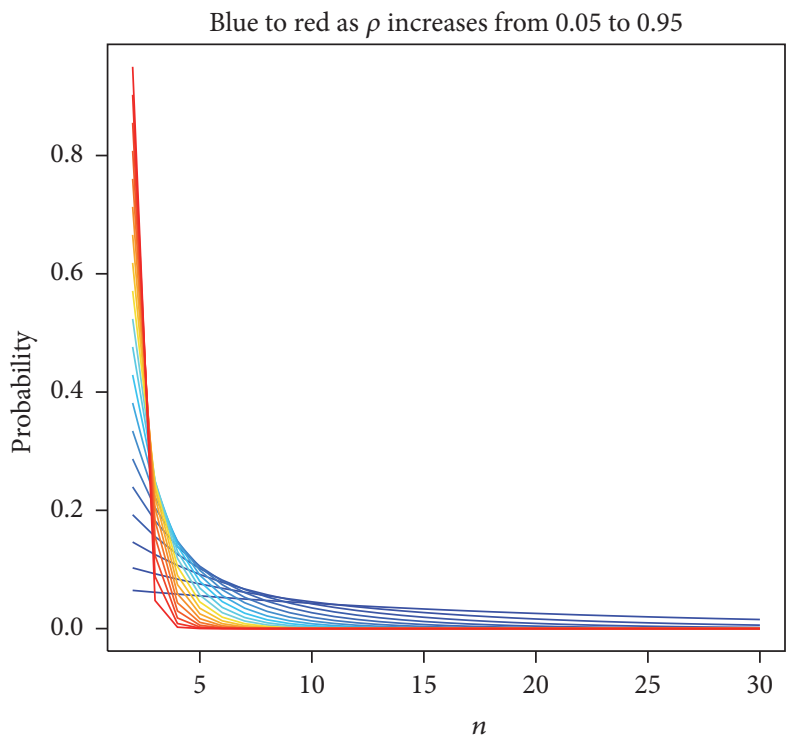

(a) Probability mass functions as $\rho$ increases, from blue $(\rho=0.05)$ to red $(\rho=1)$

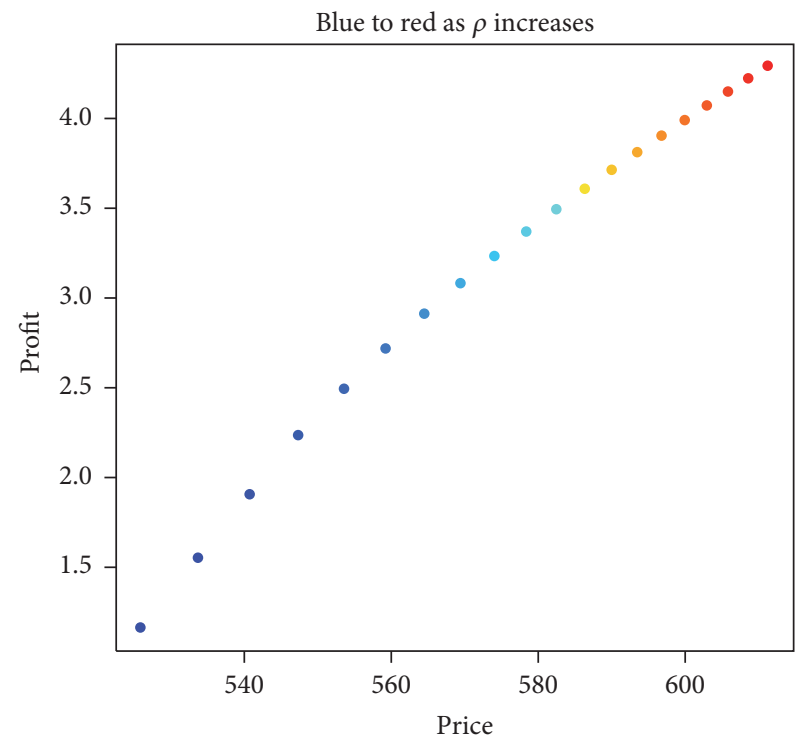

(b) Profits versus prices (optimal) for an increasing shopping fatigue ( $\rho$ increasing from blue $(\rho=0.05)$ to red $(\rho=1))$

FIGURE 4: The seller's problem under shopping fatigue. Main parameters in Table 3 and those used earlier as benchmark $\left(\mu=600, \sigma^{2}=\right.$ $125, \nu=2, \alpha_{c}=0.15, N=30$ ).

The probability mass functions for an increasing sequence of values of $\rho$, from blue $(\rho=0.05)$ to red $(\rho=1)$, can be seen in Figure 4(a). As $\rho$ decreases, the entropy of the distribution increases; in turn the shopping distribution becomes more heterogeneous.

Figure 4(b) displays the seller's response with respect to an increasing fatigue, that is, a higher propensity of the representative consumer to stop shopping in the market.
The red point corresponds to the benchmark case $(\rho=1)$ in which the consumer visits 2 shops almost surely. As the fatigue is reduced ( $\rho$ decreases) the probability of visiting more shops increases and the shopping distribution becomes more uniform. The seller reacts to the fatigue reduction by lowering prices which entails lower profits. In particular, as the shopping distribution becomes more heterogeneous, the seller's profits diminish. 
Notice that the benchmark case $\rho=1$ of Section 4.2.2 (red point in Figure 4(b)) is equivalent to the benchmark case $K=2$ of Section 4.2.1 (blue point in Figure 3(b)), and the pattern of the seller's solution is similar in both cases: as the shopping distribution becomes more heterogeneous, profits are reduced.

\section{Conclusions}

In this paper we have introduced a general framework to select the optimal pricing strategy of a seller competing in a market of a homogeneous product in which prices are dispersed and he has limited information about both his competitors' prices and the shopping behavior of his potential customers. In order to solve his problem in a real setting, the seller needs to estimate the distribution of prices in the market and the distribution of shopping behavior of consumers. By computing the solution to the problem, he determines the price that maximizes his expected profits. This theoretical setting describes many prevailing situations in real scenarios.

In general, the solution to the seller's problem depends in a delicate manner on many parametric and functional inputs. We have tested the model response under different scenarios in which some of the parameters or functions are modified. In order to analyze the seller's response under different exogenous perturbations, we have produced synthetic price data using a wide family of distributions and have generated consumer's shopping data from some naive behavioral assumptions.

Regarding the supply side of the market, a significant issue for the seller is how much (limited) information is required about his rivals' prices in order to determine his own price. In real markets it is not unusual that sellers set their prices looking only at the mean price in the market (and maybe also at some measure of price dispersion). Our computational study has showed that optimal pricing is sensitive not only to the mean and dispersion of prices but also to the shape of the distribution. Thus, in order to price efficiently, the seller must estimate the whole distribution of prices. This fact is in contrast with the behavior of the demand side of the market when shopping behavior is determined from rational time allocation. Indeed, the analysis in Alvarez et al. [6] suggested that consumers' shopping behavior can be determined from price mean and variance only, so the shape of the distribution does not seem to play a role here.

Regarding the demand side of the market, an important question for the seller is understanding how diversity in shopping behavior affects his pricing policy. In real markets, consumers' shopping behavior is typically expected to be far from rational and in turn nonhomogeneous. We have analyzed two different types of heterogeneity in the shopping distribution. Our computational analysis has showed that more diversity in the shopping behavior-parameterized, for example, by the Shannon's entropy-entails lowering optimal prices and profits.

Price distributions and shopping distributions are the main inputs to run the pricing model. The real utility of the model proposed in this paper should come from its use in real markets. It is apparent from our analysis how the model could be implemented in real markets once accurate estimates of market prices and consumer behavior are available.

\section{Competing Interests}

The authors declare that there is no conflict of interests regarding the publication of this paper.

\section{Acknowledgments}

José-Manuel Rey was partially supported by Grant AGL201569697-P from the Plan Nacional de Investigacion Cientifica y Técnica of Spain and by a Research Grant of the Real Colegio Complutense (RCC) at Harvard. This work was conceived while José-Manuel Rey was an RCC Fellow visiting the Department of Psychology at Harvard University. He thanks Professor Dan Gilbert for his warm hospitality during his stay.

\section{References}

[1] J. Bertrand, "Review of Cournot," Journal des Savants, vol. 67, pp. 499-508, 1838.

[2] M. R. Baye, J. Morgan, and P. Scholten, "Information, search, and price dispersion," in Handbook on Economics and Information Systems, vol. 1, 2006.

[3] G. Kaplan and G. Menzio, “The morphology of price dispersion," International Economic Review, vol. 56, no. 4, pp. 11651206, 2015.

[4] G. J. Stigler, “The Economics of Information,” Journal of Political Economy, vol. 69, no. 3, pp. 213-225, 1961.

[5] K. Burdett and K. L. Judd, "Equilibrium price dispersion," Econometrica, vol. 51, no. 4, pp. 955-969, 1983.

[6] F. Alvarez, J.-M. Rey, and R. G. Sanchis, "Choice overload, satisficing behavior, and price distribution in a time allocation model," Abstract and Applied Analysis, vol. 2014, Article ID 569054, 9 pages, 2014.

[7] B. Scheibehenne, R. Greifeneder, and P. M. Todd, "Can there ever be too many options? A meta-analytic review of choice overload," Journal of Consumer Research, vol. 37, no. 3, pp. 409425, 2010.

[8] R. G. Sanchis, J.-M. Rey, and F. Álvarez, "Numerical analysis of a time allocation model accounting for choice overload," International Journal of Computer Mathematics, vol. 91, no. 2, pp. 315326, 2014.

[9] B. Schwartz, "Self-determination: the tyranny of freedom," American Psychologist, vol. 55, no. 1, pp. 79-88, 2000.

[10] S. S. Iyengar and M. R. Lepper, "When choice is demotivating: can one desire too much of a good thing?" Journal of Personality and Social Psychology, vol. 79, no. 6, pp. 995-1006, 2000.

[11] B. Schwartz, A. Ward, J. Monterosso, S. Lyubomirsky, K. White, and D. R. Lehman, "Maximizing versus satisficing: happiness is a matter of choice," Journal of Personality and Social Psychology, vol. 83, no. 5, pp. 1178-1197, 2002.

[12] R Core Team, R: A Language and Environment for Statistical Computing, R Foundation for Statistical Computing, Vienna, Austria, 2014, http://www.R-project.org/.

[13] C. Forbes, M. Evans, N. Hastings, and B. Peacock, Statistical Distributions, John Wiley \& Sons, New York, NY, USA, 2011.

[14] R. M. Gray, Entropy and Information Theory, Springer, New York, NY, USA, 1990. 


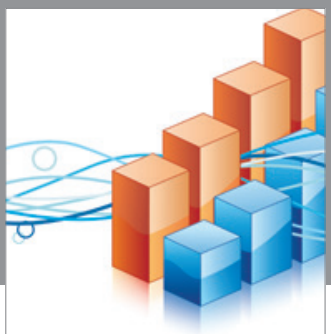

Advances in

Operations Research

vatem alat4

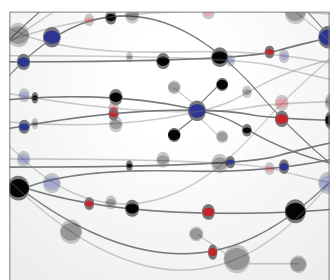

\section{The Scientific} World Journal
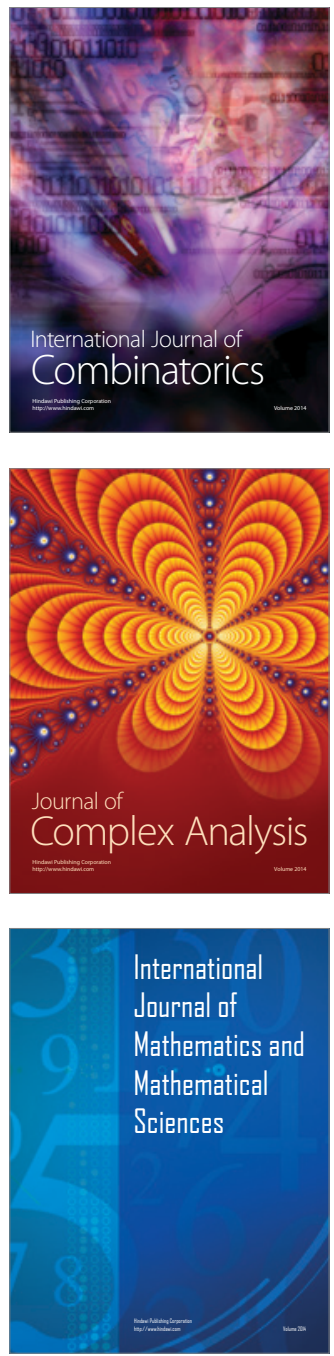
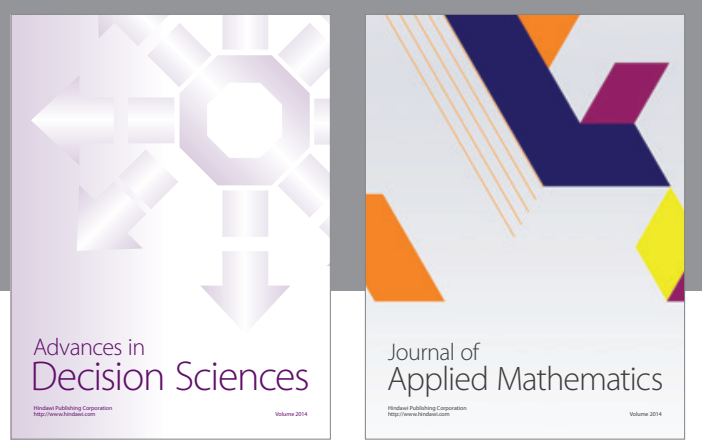

Algebra

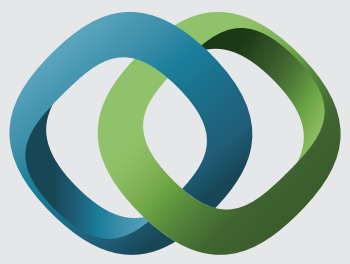

\section{Hindawi}

Submit your manuscripts at

http://www.hindawi.com
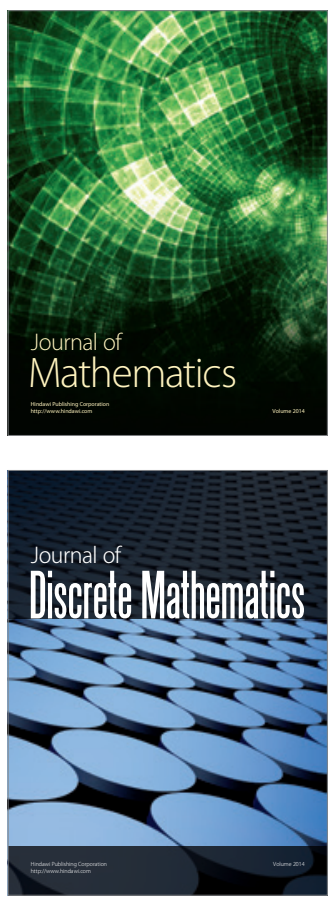

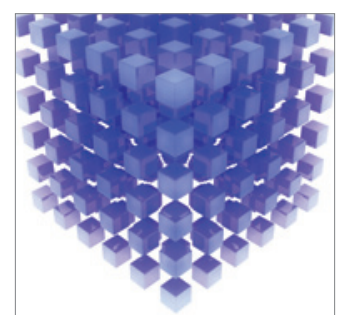

Mathematical Problems in Engineering
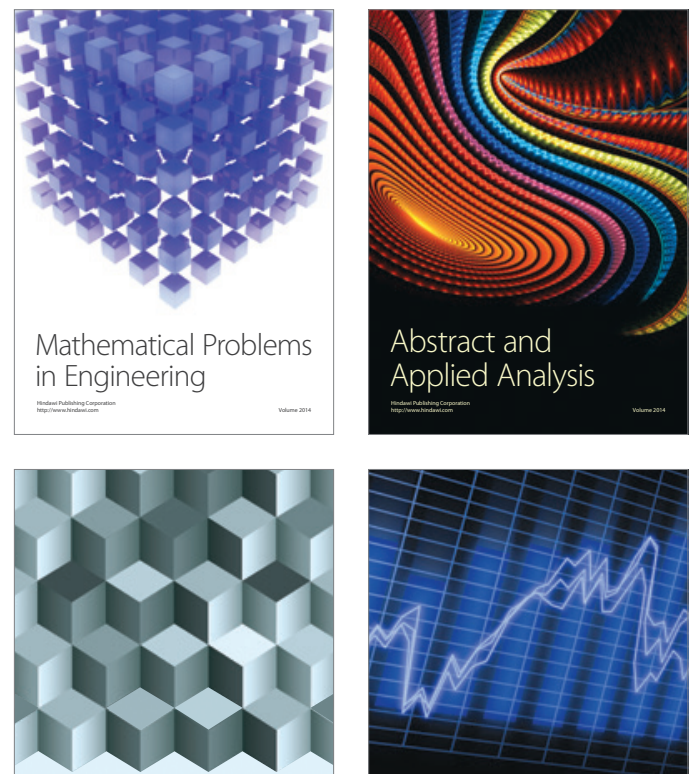

Journal of

Function Spaces

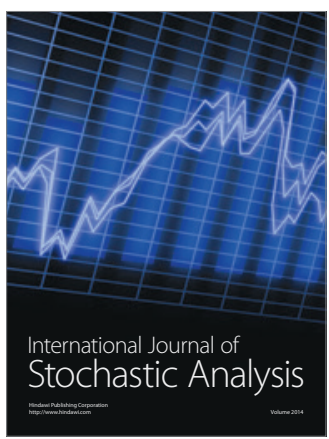

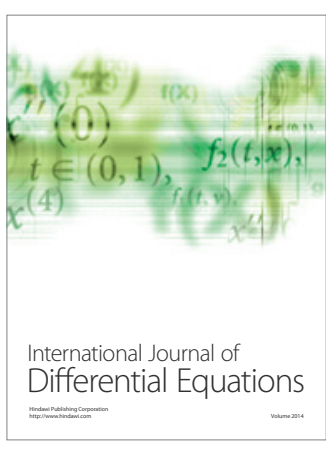
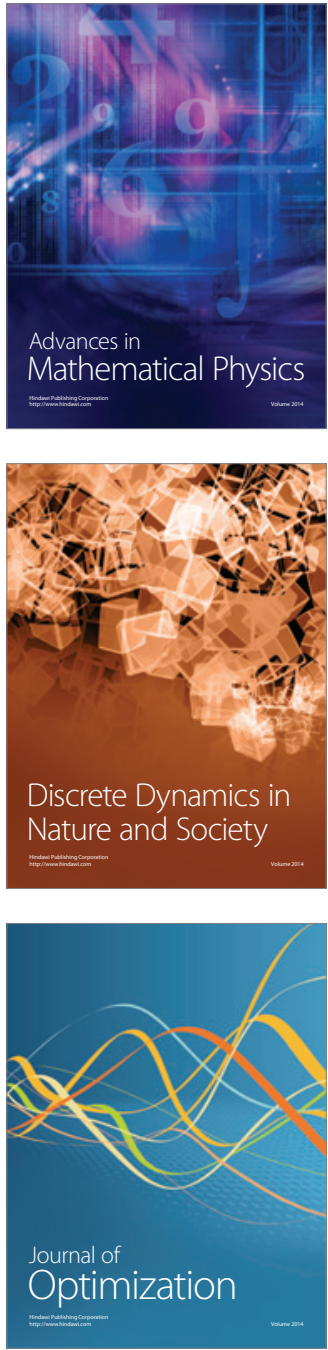